\title{
MEASURED Ni-63 CONTENTS IN SAVANNAH RIVER SITE HIGH LEVEL WASTE AND DEFENSE WASTE PROCESSING FACILITY GLASS PRODUCT BY Ni-SELECTIVE ION EXCHANGE PURIFICATION AND $\beta$-DECAY COUNTING (U)
}

\author{
R. A. Dewberry, N. E. Bibler, and D. P. DiPrete \\ Savannah River Technology Center \\ Westinghouse Savannah River Company, \\ Aiken, SC 29802., USA
}

\begin{abstract}
In this paper we describe tests of EiChrom Industries' Ni-selective ion exchange resin for use in analysis of Ni-63 in Savannah River Site high level waste. We report measurement of Ni-63 content in two sets of Savannah River Site glass product from the Defense Waste Processing Facility.

The Ni-63 $\beta$-decay activity was chemically separated in quintuplicate from fission product and plutonium $\alpha-\beta$ activities of up to $10^{3}$ times the observed Ni-63 content. The separation used a Ni-dimethlyglyoxime precipitation followed by radiochemical purification using the Ni-selective extraction chromatography resin. Further removal of interfering activity was based on diagnosis of observed radioactivity in each successive product phase. We analyzed eleven plant glass product samples using seven spiked standard addition duplicates to measure Ni-63 recovery in the separations. Selected liquid scintillation $\beta$-decay spectra are shown to validate the method. Interpretation of accuracy is based upon three distinct comparisions to predicted Ni-63 content.
\end{abstract}

\section{INTRODUCTION}

Ni-63 analysis is an important measurement at the Savannah River Site (SRS) because it is required in the Defense Waste Processing Facility (DWPF) glass product waste acceptance criteria ${ }^{1}$. These criteria require we report the content of any radionuclide which will represent as much as $0.05 \%$ of the total activity of the glass at any time in the first 1100 years of isolation. With half-lives of 100 years and 75000 years, both Ni-63 and Ni-59 satisfy this requirement even though their initial activity in SRS waste represent only a tiny fraction of the total activity (far less than the threshold $0.05 \%$ of total). Neither species can be measured directly by ICP-MS because of dominant isobaric interferences, and so we require a chemical separation to isolate their $\beta$-decay activities to measure Ni-63 content. The chemical separation to measure Ni-59 by ICP-MS or by $\mathrm{x}$-ray pulse height analysis is very similar, though we do not address that analysis in detail in this paper.

Previous work at the Savannah River Technology Center (SRTC) to obtain a purified nickel fraction was based on the precipitation of Ni-dimethylglyoxime (Ni-DMG) followed by successive liquid-liquid extractions. ${ }^{2-4}$ This technique has been successful to report Ni-63 content in SRS high level waste, but is quite labor intensive, uses a suspected carcinogen $\left(\mathrm{CH}_{2} \mathrm{Cl}_{2}\right)$, and produces approximately $25 \mathrm{ml}$ of radioactive acidic and organic waste per sample analysis. Other development work at SRTC has involved the use of ion chromatography coupled with either liquid scintillation counting or ICP-MS to measure the purified Ni fractions ${ }^{5}$. This technique has produced limited success with SRS high level waste feed to the DWPF, but has not produced a purified nickel fraction suitable for measure of actual $\mathrm{Ni}-59$ events by mass spectroscopy or of actual Ni-63 events by liquid scintillation spectroscopy. 
In this paper we describe development work for purification of a Ni-63 fraction by column extraction chromatography using EiChrom Industries' Ni-selective resin ${ }^{\mathrm{TM}}$. After testing with four cold samples and nine radioactive simulant samples, we report measurement of Ni-63 content in two sets of eleven SRS high level waste glass product samples from the DWPF. The chemical separation used a Ni-DMG precipitation pretreatment followed by radiochemical purification using the EiChrom Industries Ni-selective extraction chromatography resin. Further removal of interfering activity was based on diagnosis of observed radioactivity in each successive intermediate phase. This technique of purification by successive diagnostic testing has replaced the labor intensive precipitation and liquid-liquid extraction procedure previously in use at SRTC. Since neither Ni-63 nor Ni-59 is a fission product, but instead are neutron activation products, their radioactivity in SRS high level waste is dominated by other fission products and by neutron activation Pu activity. The Ni-63 $\beta$-decay activity was chemically separated in quintuplicate from fission product and plutonium $\alpha-\beta$ activities of up to $10^{5}$ times the observed Ni-63 content. Ni-63 chemical recoveries were measured from quadruplicate spiked standard addition aliquots and were near $50 \%$ with $15 \%$ RSD precision at the end of a six step "procedure".

Our initial tests of the Ni-selective resin using only a load and elute step as prescribed by the vendor indicated we could obtain good recovery of elemental nickel and good purification from selected species measured by ICP-AES. However further tests with spiked radioactivity demonstrated that the single step extraction would not attain adequate purification for our purposes of Ni-63 from Cs-137, Pu, or Tc-99. The tests with spiked activity did indicate good purification of Ni-63 from Sr-90. By adding a Ni-DMG precipitation pretreatment we were able to demonstrate good purification of Ni-63 from Cs-137, C-14, and Sr-90, but we did not observe significantly improved purification from Pu or Tc-99. Since Pu-238,239,240 alpha activity in SRS waste is always accompanied by Pu-241 beta activity (end point 21keV), Pu in our Ni product phases constituted an unacceptable interference in determination of Ni-63 ( $\beta$ decay endpoint $66 \mathrm{keV})$.

In subsequent analyses of actual glass product samples from the DWPF, we treated eleven samples and seven spiked standard addition aliquots in two sets of analyses. We obtained liquid scintillation spectra of each nickel product phase after the precipitation and column extraction step. From the spectra we were able to diagnose interference from Sr-90/Y-90, Cs-137, and Pu $\alpha$-activity. All of the samples and standard addition aliquots were then treated in separate steps for Cs-137 removal by ion exchange using Cs-selective AMP-1 resin, for Sr-90 removal by extraction chromatography using Sr-selective Sr Spec resin, and for Pu removal by extraction chromatography using TRU Spec resin. Each successive product phase spectrum was observed to determine that it was pure of the dominant offending activity, until we finally obtained spectra in which we observed authentic, pure Ni-63 events. $\mathrm{Ni}-63$ recovery was then measured on the spiked aliquots. We obtained average recovery and precision which were applied to the analyses of the unspiked samples. Measured values and selected product phase liquid scintillation $\beta$-decay spectra are shown to validate the measurements. Our discussion of estimated accuracy involves comparison with other more easily measured radionuclides using theoretical calculations and also using some more complex arguments involving DWPF process knowledge. This discussion is presented in a separate section below. 


\section{EXPERIMENTAL}

\section{Initial Tests}

Our first test of the EiChrom Ni-selective resin involved a test with four cold samples in which we loaded each sample onto the pre-packed vendor columns in 88:1-diluted concentrated $\mathrm{NH}_{4} \mathrm{OH}$ and then eluted with $4 \mathrm{M} \mathrm{HNO}_{3}$. The product phases and the load eluates were each measured for nickel content using ICP-AES. We observed no nickel in any of the load eluate phases, and we obtained recoveries of $89 \%, 86 \%, 87 \%$, and $60 \%$ in the four product phases. We then ran a set of three samples spiked with a mix of radioactivity including Cs-137, Tc-99, Ni-63, Sr-90, C-14, and $\mathrm{Pu}$-238. The product phases of the two-step load-elute experimental technique demonstrated we obtained near $100 \%$ recovery of spiked Ni-63, but we also observed interference in the product phases from Tc-99, Pu-238, and Sr-90. The liquid scintillation spectra of each of these three product phases is shown in Figure 1. Recovery of Tc-99 is about $50 \%$ and of Pu-238 is about $10 \%$. Recovery of spiked Sr-90 in the first sample is very small, but even a tiny fraction would represent a significant interference in actual SRS high level waste.

We performed a second set of separations using six samples spiked with the radioactivity listed in Table 1. In this experiment we included a pretreatment step intended to remove the interferences noted above. We prepared the samples in 10:1-diluted $\mathrm{NH}_{4} \mathrm{OH}$ and precipitated each with $1 \% \mathrm{DMG}$ in ethanol. The pink precipitates were rinsed with dilute $\mathrm{NH}_{4} \mathrm{OH}$ and were then dissolved with $4 \mathrm{ml}$ of $2 \mathrm{M} \mathrm{HCl}$. The solutions were back titrated to slightly basic with dropwise addition of concentrated $\mathrm{NH}_{4} \mathrm{OH}$. The basic products were then loaded onto the Ni-selective extraction columns and subsequently eluted with $3 \mathrm{M} \mathrm{HNO}_{3}$ as above. The liquid scintillation spectra of four of the six product phases are shown in Figure 2. In the six we attained 21(8)\% recovery of spiked Ni-63. $\gamma$-PHA data demonstrated $100 \%$ purification from Cs-137. In the liquid scintillation spectra we observed $100 \%$ purification from C-14 and Sr-90 (though there is measurable $\mathrm{T}_{1 / 2}=64$-hour Y-90). However it is clear in the spectra that for every sample which contained Pu-238, we obtained a Pu-238 component in the product, and for the three samples which contained spiked Tc-99, we observed 3(2)\% recovery of it. We concluded that the EiChrom resin would be useful with some adaptation to measure Ni-63 in actual DWPF sludge-only feed and sludge-only glass product ${ }^{6}$.

\section{Analyses of Actual DWPF Product}

With the encouraging results above we attempted an analysis of $\mathrm{Ni}-63$ content in a set of six actual sludge-only DWPF glass product samples. These measured values represented an important analysis, because the samples were from the very first batch of product glass to come from the DWPF plant. These analyses would represent SRTC's only mechanism to report Ni-63 content to satisfy the waste acceptance criteria ${ }^{1}$ for this batch of glass. The reported values could be checked against the predicted value calculated from the measured $\mathrm{Ni}-63$ content in the DWPF sludge-only feed ${ }^{7,8}$ to gain confidence in the measurement. The technique of this check is described below and in reference 7 . 
The batch of six samples SRTC received from the DWPF were dissolved with an HCl/HF dissolution, and were assigned the laboratory information system (LIMS) numbers $85840, \ldots 85845$. Samples 85844 and 85845 were blanks. The other four had been previously analyzed to contain Sr-90 at approximately $10^{6} \mathrm{dpm} / \mathrm{ml}$, Pu- $\alpha$ at $10^{5}$ $\mathrm{dpm} / \mathrm{ml}$, Pu-241 near $2 \times 10^{4} \mathrm{dpm} / \mathrm{ml}$, and Cs-137 at about $4000 \mathrm{dpm} / \mathrm{ml}$. The Tc-99 content was not measured, but was expected to be below $8000 \mathrm{dpm} / \mathrm{ml}$. We obtained $1 \mathrm{ml}$ aliquots of each of the six samples and duplicate aliquots of samples 85841,85843 , and 85844 which we spiked with $0.475 \mu \mathrm{Ci}$ of Ni-63 standard from a decay corrected source which had been vendor certified in December 1989. All nine were treated by DMG precipitation and were then loaded onto the Ni-resin and eluted as prescribed above. We obtained liquid scintillation spectra of these product phases to observe interferences. The spectra of three product phases denoted with the prefix $\mathrm{R}$ are shown in Figure 3 , along with that of sample Y85859 which we discuss below. We appear to attain good and measurable recovery of spiked Ni-63 in phase R85841.sp and in the other two spiked samples, but there clearly is significant interference from $\mathrm{Sr}-90 / \mathrm{Y}-90$ and $\mathrm{Pu}-\alpha$ activity in all nine of these intermediate phases.

We added $1000 \lambda$ of each of these R-phases to $3 \mathrm{ml}$ of $4 \mathrm{M} \mathrm{HNO}_{3}$ and passed the $4 \mathrm{ml}$ composite through a mixed bed extraction chromatography column consisting of Pu-selective EiChrom TRU Spec resin and of EiChrom Sr-selective $\mathrm{Sr}$ Spec resin. The $4 \mathrm{M} \mathrm{HNO}_{3}$ matrix was already ideal for loading the $\mathrm{Pu}$ and $\mathrm{Sr}$ onto their selective resins. The nickel in each of the nine samples passed through the resins into the load eluate phases which we labeled with the convention T85840,... T85845. We eluted the Pu and $\mathrm{Sr}$ from the columns with rinses of $0.01 \mathrm{M}$ oxalic acid in $0.05 \mathrm{M}$ $\mathrm{HNO}_{3}$ and of $0.0001 \mathrm{M} \mathrm{HNO}_{3}$ and found we were able to regenerate the mixed bed columns for multiple use.

Selected spectra of the intermediate T-phases are shown in Figure 4. The spectra were observed to be extremely pure of Sr-90 and of Pu- $\alpha$ activity, which was exactly the intention of the mixed bed columns. At time $t=0$ each spectrum had a dominant 2.2-MeV endpoint $\mathrm{Y}-90$ component. After about 17 days, these components had decayed to below background levels in all but two of the samples, but there remained a lower energy component at about 30 $\mathrm{dpm} / \mathrm{ml}$ in seven samples, which would not decay. This interference was determined to be Cs-137 activity.

We performed a Cs-removal by adding about $1 / 2$ gram of Cs-selective AMP-1 resin directly to these $4 \mathrm{M} \mathrm{H}^{+}$samples. We stirred and let the resin settle, and in this way the Cs-removal did not introduce any dilution to the product phases. We obtained liquid scintillation spectra of these product phases labeled with the convention T85840-Csr,...T85845Csr. These spectra appeared to be pure of any events except 66-keV endpoint Ni-63. Three T-phase Cs-removal spectra are shown in Figure 5. The measured count rates taken from $500 \lambda$ aliquots of each T-...-Csr product phase are tabulated in Table 2. The overall dilution factor for each sample treatment was 37.9. In column three of Table 2 we list the scintillation counting efficiency determined by the Packard tSIE method ${ }^{9}$. We determined a recovery of $20(10) \%$ from the three spiked samples, which we applied to all of the other six samples to determine measured $\mathrm{Ni}-63$, listed in column 5 . We finally reported a composite measured value of $[\mathrm{Ni}-63]=7600(800) \mathrm{dpm} / \mathrm{ml}$. Our interpretation of accuracy is discussed below.

We then repeated the measurement on a set of five glass samples which we prepared with an initial dissolution by sodium peroxide fusion. These samples were assigned the LIMS numbers $85857, \ldots 85861$ and were prepared with the identical DMG precipitation, back titration to slightly basic, and load-elute sequence as described above. For this set of samples we prepared four spiked duplicates labeled 85858.sp through 85861.sp which received a decay corrected spike of $0.237 \mu \mathrm{Ci}$ of $\mathrm{Ni}-63$ standard. 
As above, the intermediate, diagnostic product phases had interferences from Sr-90/Y-90, Pu- $\alpha$, and Cs-137 activity. No Tc-99 interference was observed in this sludge-only glass. Spectrum Y85859 in Figure 3 shows a huge interference from Sr-90/Y-90 and Pu- $\alpha$ in the first elution phase of that sample. Figure 6 shows two spectra from the next product phases after Sr and Pu removal. The lower spectrum was taken after several days of decay, but where the Y-90 component is still obvious, and the upper spectrum was taken after several more days of decay, where the Y-90 component is no longer discernable. In both spectra the Ni-63 component is easily observable. In Table 3 we have recorded the observed count rates, recoveries, counting efficiencies, and measured values for these five samples and four spiked duplicates obtained after complete purification. The complete purification product phases bear the prefix B in Table 3. The overall average recovery was 50(8)\%. The larger value and improved precision we believe were due to a combination of better laboratory technique and use of the $\mathrm{Na}_{2} \mathrm{O}_{2}$ fusion initial dissolution. We reported a composite content of $4300(600) \mathrm{dpm} / \mathrm{ml}$ for these five DWPF glass product samples.

\section{INTERPRETATION OF ACCURACY}

Interpretation of our accuracy is based on reactor code calculations which allowed us to predict approximate expected ratios between Ni-63 content and the important fission product Sr-90 and between Ni-63 content and Np-237 content, Pu-238,239 content and Am-241 content ${ }^{10}$. Note that the last four are actinide neutron activation products. We do not use the easily measured Cs-137 content, because it is very difficult to predict the Cs-137 content in the sludge component of SRS high level waste. Another check on our accuracy is based on the expected ratios between measured Ni-63 in the DWPF feed and measured Ni-63 in the DWPF glass product ${ }^{7,8}$. The Ni-63 sludge-only feed content was measured and reported by the full precipitation, liquid-liquid extraction technique in previous use at $\mathrm{SRTC}^{4}$. A third measure of accuracy can be taken from the ratios of Ni-63/Ni-59 activity. An upper limit of Ni-59 content in the sludge-only feed was obtained by separate purification and measure by ICP-MS. Ni-59 content in the glass product was measured by $x$-PHA on the identical product phases of this paper. Thus these three data combined provide a good opportunity to check the accuracy and self-consistency of the Ni-63 measurement techniques.

Using fission product abundances, known fuel and cladding content, and estimated neutron flux and fluences, Baxter $^{10}$ was able to calculate predicted radionuclide contents for DWPF sludge-only feed and sludge-only glass. While this calculation contains many variable parameters, we believe it provides a reliable estimate of activity ratios expected for the individual radionuclide contents of DWPF feed and glass samples. The predicted ratios of Ni-63 activity relative to five easily measured species are shown in Table 4 along with our measured ratios for each of these species. In three cases the calculated estimates differ from our measured ratios by a value greater than what we believe to be our measurement uncertainty. However, we believe the agreement is sufficient to establish good credibility in our Ni-63 measured values. 
For the second test of accuracy, the calculation of expected ratio of Ni-63 content in the DWPF feed and glass product is based on the waste dilution factor method developed and described in references 7 and 8 . We observe that by measuring the concentrations of nonradioactive $\mathrm{Fe}, \mathrm{Al}, \mathrm{Mn}$, and $\mathrm{Ca}$ and the concentrations of several easily measured radioactive species (eg. Sr-90, Cs-137, U-238, and Am-241) in the feed and glass product, a waste dilution factor (WDF) of $3.07 \pm 0.21$ can be calculated. This WDF for a given species represents the ratio of measured content of that species in the feed to its content in the glass product. Applying this WDF to all of the other 44 more difficult to measure radioactive species, we have found that 28 out of the 44 ratios agreed to within $30 \%$ of the predicted 3.07. Only two of the 44 ratios were off by a factor of greater than 2 . Several of the measured sludge to glass ratios are shown plotted in Figure 7 . The value obtained for Ni-63 content including uncertainty is $(2.65 \pm 0.81)$, in good agreement with the predicted ratio. We believe it bears repeating that the DWPF feed and glass product $\mathrm{Ni}-63$ contents were measured after two distinct separation techniques.

In our third test of accuracy, we were not successful to obtain a measured value for Ni-59 content in the DWPF feed samples using separation followed by ICP-MS analysis. The upper limit of content we measured was two orders of magnitude above the expected value. However for the glass product samples, we counted the purified Ni product phases by x-ray pulse height analysis using a low energy photon (LEP) detector to observe the electron capture Co-59 daughter $6.9 \mathrm{keV} \mathrm{K}_{\alpha} \mathrm{x}$-ray doublet. Since Ni-63 decays in the opposite atomic number direction, its $\mathrm{Cu}-63$ daughter $8.0 \mathrm{keV}$ x-ray doublet does not interfere. Several of the Ni product phases were counted for Ni-59 content by preparing a $1 \mathrm{ml}$ evaporated plate. This allowed us to closely couple the plate to the planar LEP detector. Counting the direct liquid sample is not practical since the $6.3 \mathrm{keV} \mathrm{x}$-ray would be strongly absorbed by a container vial. Even in the plate configuration, detection efficiency is only about $0.1 \%$. The $\mathrm{K}_{\alpha} \mathrm{x}$-ray branch is only $20 \%$.

Due primarily to the low detection efficiency, we were able to measure only an upper limit of about $4000 \mathrm{dpm} / \mathrm{ml}$ of $\mathrm{Ni}-59$ activity in the second set of glass samples. Our best measured ratio of Ni-59/Ni-63 is then $<0.9$. The value predicted by Baxter 10 is 0.0082 , again about two orders of magnitude below our detection limit. Therefore our third test of accuracy for Ni-63 content yields no result beyond observing that the measured ratio does not contradict our prediction.

Two of the three comparisons of measured Ni-63 content to theoretical predictions lend good credibility to the accuracy of our measurements, and the third comparison supports our measurement. 


\section{CONCLUSION}

We have demonstrated the capability to separate a pure fraction of $\mathrm{Ni}$ suitable to measure $\mathrm{Ni}-63$ content in high level waste samples whose Sr-90 content and Pu- $\alpha$ and Pu-241 $\beta$-decay content is up to three orders of magnitude larger than the Ni-63 we wish to measure. The Ni-63 measurement fullfills one of the required analyses of the Defense Waste Processing Facility waste acceptance criteria. Our technique is based upon a Ni-dimethylglyoxime precipitation pretreatment followed by Ni-selective extraction chromatography. Subsequent treatment is based upon selective ion exchange and extraction chromatograhy to remove diagnosed interfering activity. Thus simple Niselective extraction using commercially available resin alone does not provide an adequate purification for our purposes. However our combination of selective extraction with diagnostic testing and sample-specific treatment has been successful.

The method we use is much less labor intensive than the precipitation and liquid-liquid extraction procedure in previous use at the Savannah River Technology Center. Our technique also greatly reduces radioactive waste, particularly organic waste, and avoids the use of carcinogenic $\mathrm{CH}_{2} \mathrm{Cl}_{2}$, which the previous procedure prescribes. Our method of separation has not followed a set procedure intended for use on all samples, but rather uses diagnostic testing to apply treatments to remove interfering activity as it is observed in each intermediate product phase. This allows the chemist to shortcut unnecessary steps that might occur in a standard procedure.

We have used triplicate and quadruplicate standard addition samples to measure recovery and to bias correct in the two sets of actual DWPF glass product samples that we have analyzed, and we have used standard tSIE efficiency correction when counting the product phases by liquid scintillation spectroscopy. Variance in the standard addition recoveries has been the largest contributor to our measurement uncertainty. We have been able to check the accuracy of the measurement using three comparisons which we describe in the text. Since one of the comparisons involves measurement of Ni-63 using the previous separation procedure, we were able to obtain a measure of the self-consistency of the two separation techniques. 


\section{REFERENCES}

1. Office of Environmental Restoration and Waste Management, Waste Acceptance Product Specifications for Vitrified High-Level Waste Forms, USDOE Document EM-WAPS, U.S. Department of Energy, Germantown, MD, 1993.

2. J. Kleinberg and H. L. Smith, Collected Radiochemical Procedures (Radiochemistry Group CNC-11), Los Alamos Scientific Laboratory, LA-1721, 4th Ed.

3. C. J. Coleman, "Separation of Nickel From Fission Products", Approved ADD Procedures, Savannah River Laboratory Internal Communication, December 1982.

4. R. A. Dewberry, "DWPF Radiochemical Analytical Procedures for Glass Product Acceptance," WSRC-RP-94-566-TL, Savannah River Technology Center Internal Communication, June 1994.

5. A. C. Almon, W. F. Kinard, R. A. Dewberry, and N. E. Bibler, Multimodal Separation of Alkali, Alkaline Earth, Transition, Post-Transition, and Actinide Cations in Waste Sludge, Symposium on Ion Chromatography for the Power Industry, New Orleans, LA, May 1991.

6. R. A. Dewberry, SRT-ADS-96-0304, Savannah River Site Internal Communication, July 1996.

7. W.F. Kinard, N.E. Bibler, C. J. Coleman, and R. A. Dewberry, J. Radioanal. Nucl. Chem. 219 (1997) 197.

8. N. E. Bibler, W. F. Kinard, R. A. Dewberry, and C. J. Coleman, WSRC-TR-94-0505, Savannah River Site Internal Communication, October 1994.

9. Y. Kobayashi, Liquid Scintillation Analysis, Packard Instrument Co, 1988.

10. Baxter DP-1606, Savannah River Plant Internal Communication, December 1988.

11. R. A. Dewberry, "DWPF Repository Analyses," Savannah River Laboratory Internal Communication, September 1986. 
Table 1. Spiked radioactive contents used in the second set of development samples described in the text. Each sample also received a spike of $22000 \mathrm{dpm} \mathrm{Ni}-63$.

$\begin{array}{llll}\text { Sample 92063: } & 54300 \mathrm{dpm} \text { Pu-238 } & \text { Sample 92064: } & 24000 \mathrm{dpm} \text { Tc-99 } \\ \text { Sample 92065: } & \begin{array}{l}76000 \mathrm{dpm} \text { C-14 } \\ 50 \lambda \text { SRS HLW }\end{array} & \text { Sample 92066 } & 2000 \mathrm{dpm} \text { Co-60 } \\ & 2000 \mathrm{dpm} \text { C0-60 } & & 54300 \mathrm{dpm} \text { Pu-238 } \\ & 10000 \mathrm{dpm} \text { Sr-90 } & & 50 \lambda \text { SRS HLW } \\ 24000 \mathrm{dpm} \text { Tc-99 } & & 24000 \mathrm{dpm} \text { Tc-99 } \\ & & \text { Sample 92068: } & 50 \lambda \text { SRS HLW } \\ & & & 10000 \mathrm{dpm} \text { Sr-90 } \\ \text { Sample 92067: } & 54300 \mathrm{dpm} \text { Pu-238 } & & 76000 \mathrm{dpm} \text { C-14 } \\ & & & 2000 \mathrm{dpm} \text { Co-60 }\end{array}$

Table 2. Measured activities, recoveries, and adopted Ni-63 contents of the T-phase product phases after final Cs-137 removal of the DWPF glass samples which received the laboratory information system numbers 85840 through 85845 . The dilution factor is 37.9 .

$\begin{array}{lllll}\text { Product Phase } & \text { cpm/0.5ml } & \text { Efficiency } & \begin{array}{c}\text { Recovery } \\ (\%)\end{array} & \begin{array}{c}{[\mathrm{Ni}-63]} \\ (\mathrm{dpm} / \mathrm{ml})\end{array} \\ & & & & 7600(3900) \\ \text { T85840-Csr } & 18(2) & 0.90 & & 8600(4700) \\ \text { T85841-Csr } & 20(2) & 0.88 & & \\ \text { T85841.sp-Csr } & 1742(9) & 0.89 & 14(1) & 7100(3900) \\ \text { T85842-Csr } & 16(2) & 0.90 & & 6900(3900) \\ \text { T85843-Csr } & 16(2) & 0.90 & & \\ \text { T85843.sp-Csr } & 1869(10) & 0.89 & 15(1) & <2000 \\ \text { T85844-Csr } & <6 & 0.91 & & \\ \text { T85844.sp-Csr } & 3996(14) & 0.90 & 32(1) & <6000 \\ \text { T85845-Csr } & 13(2) & 0.90 & & \end{array}$


Table 3. Measured activities, recoveries, and adopted Ni-63 contents of the B-phase product phases of the DWPF glass samples which received the laboratory information system numbers 85857 through 85861 . Dilution factor is 10.8 .

$\begin{array}{lllll}\text { Product Phase } & \mathrm{cpm} / \mathrm{ml} & \text { Efficiency } & \begin{array}{c}\text { Recovery } \\ (\%)\end{array} & \begin{array}{c}{[\mathrm{Ni}-63]} \\ (\mathrm{dpm} / \mathrm{ml})\end{array} \\ & & & & \\ & & & & 3500(600) \\ \text { B85857 } & 140(5) & 0.863 & & 4000(600) \\ \text { B85858 } & 160(7) & 0.872 & & \\ \text { B85858.sp } & 25600(100) & 0.863 & 60(1) & 4700(800) \\ \text { B85859 } & 192(7) & 0.874 & & \\ \text { B85859.sp } & 22000(200) & 0.872 & 52(1) & 4900(800) \\ \text { B85860 } & 197(7) & 0.873 & & \\ \text { B85860.sp } & 17300(100) & 0.872 & 41(1) & <4000 \\ \text { B85861 } & <100 & 0.843 & & \\ \text { B85861.sp } & 19200(100) & 0.854 & 46(1) & \end{array}$

Table 4. Measured and predicted ratios between Ni-63 activity and that of the fission product Sr-90 and that of several actinide neutron activation products. The predicted values are taken from references 10 and 11.

$\begin{array}{lcc}\text { Species } & \text { Measured Ni-63/Measured Species } & \text { Predicted Ni-63/Predicted Species } \\ \text { Sr-90 } & 0.0060(15) & 0.0062 \\ \text { Np-237 } & 300(100) & 333 \\ \text { Pu-238 } & 0.054(18) & 0.002 \\ \text { Pu-239 } & 0.68(27) & 0.228 \\ \text { Am-241 } & 0.48(19) & 0.27\end{array}$




\section{FIGURE CAPTIONS}

Figure 1. Liquid scintillation $\beta$-decay spectra from the first scoping test with radioactivity. Each spectrum shows a strong component from $\mathrm{Ni}-63$ in the (lowest energy) window, however each shows significant Tc-99 component in the second energy window. The last two spectra show components from $\mathrm{Sr}-90 / \mathrm{Y}-90$ and from $\mathrm{Pu}-238$.

Figure 2. Liquid scintillation $\beta$-decay spectra from selected product phases from the samples of Table 1 . Note good recovery of $\mathrm{Ni}-63$, and good purification from spiked Co-60, Sr-90, and C-14, but interference from Tc-99 and $\mathrm{Pu}-238$.

Figure 3. Liquid scintillation $\beta$-decay spectra from three intermediate phases of the first set of actual DWPF glass product samples and from one intermediate phase of the second set. The spectra show significant interferences from Sr-90/Y-90 and from Pu $\alpha$-activity.

Figure 4. Liquid scintillation $\beta$-decay spectra from four intermediate T-phases as described in the text. The spectra each show a low energy Ni-63 component and a long-lived higher energy component attributed to Cs-137.

Figure 5. Liquid scintillation $\beta$-decay spectra from three final product phases of the first set of glass samples. The spectra each show only a low energy Ni-63 component.

Figure 6. Liquid scintillation $\beta$-decay spectra from two final product phases of the second set of actual DWPF glass product samples. The spectra each show a low energy Ni-63 component and a rapidly decaying $\mathrm{Y}-90$ component.

Figure 7. Sludge to glass waste dilution factors for four stable elements and for $\mathrm{Ni}-63$ and eight other easily measured radionuclides. 
68171

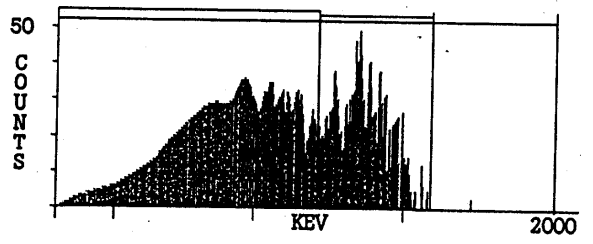

68172

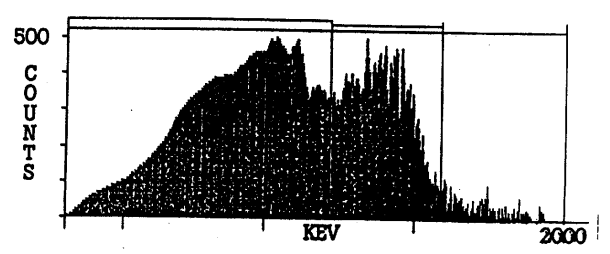

\section{3}

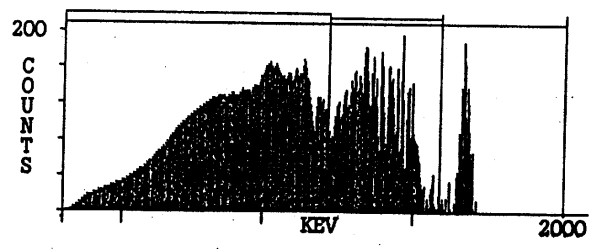

Figure 1. Liquid scintillation $\beta$-decay spectra from the first scoping test with radioactivity. Each spectrum shows a strong component from Ni-63 in the (lowest energy) window, however each shows significant TC-99 component in the second energy window. The last two spectra show components from $\mathrm{Sr}-90 / \mathrm{Y}-90$ and from $\mathrm{Pu}-238$. 
92064

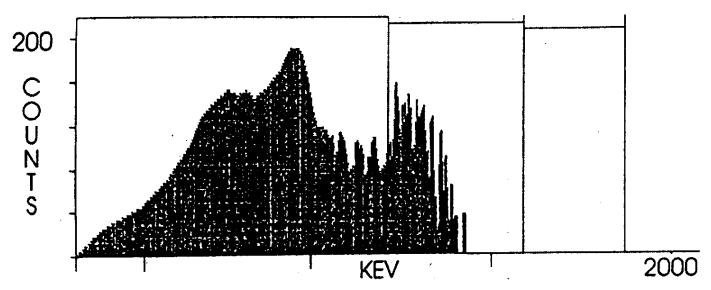

\section{7}

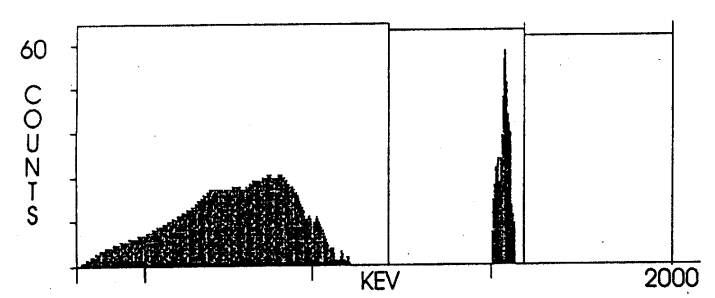

92065

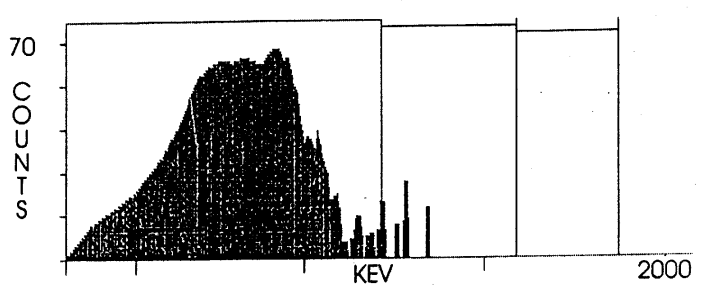

\section{8}

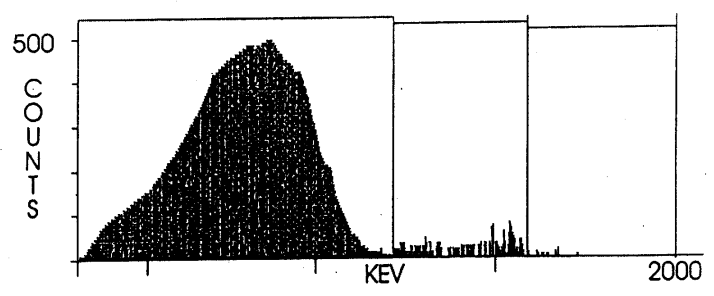

Figure 2. Liquid scintillation $\beta$-decay spectra from selected product phases from the samples of Table 1 . Note good recovery of $\mathrm{Ni}-63$, and good purification from spiked Co-60, Sr-90, and C-14, but interference from $\mathrm{Tc}-99$ and $\mathrm{Pu}-238$. 
R85841
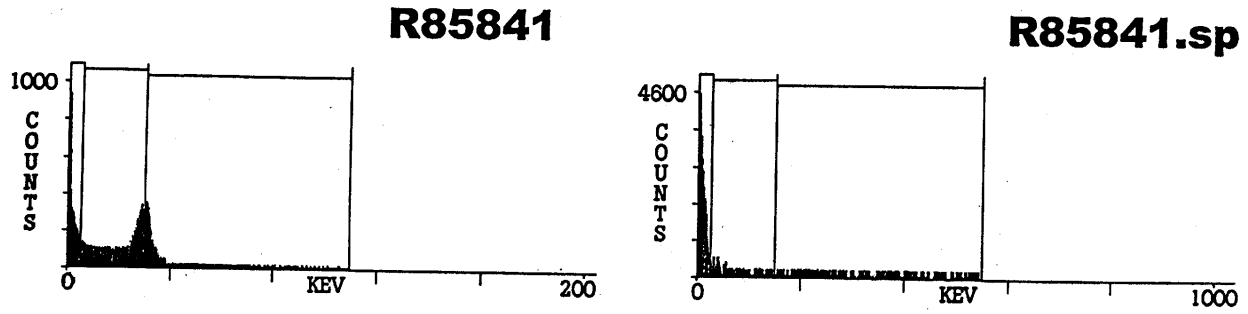

R85843

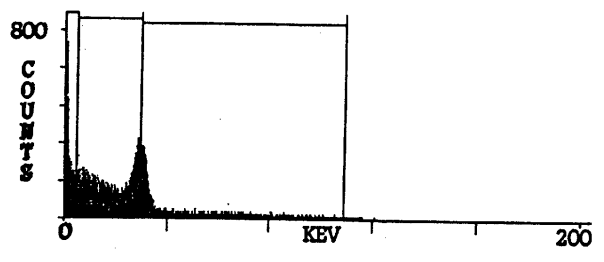

Y85859

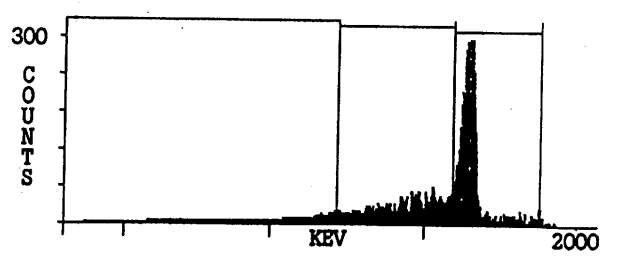

Figure 3. Liquid scintillation $\beta$-decay spectra from three intermediate phases of the first set of actual DWPF glass product samples and from one intermediate phase of the second set. The spectra show significant interferences from Sr-90/Y-90 and from Pu $\alpha$-activity. 

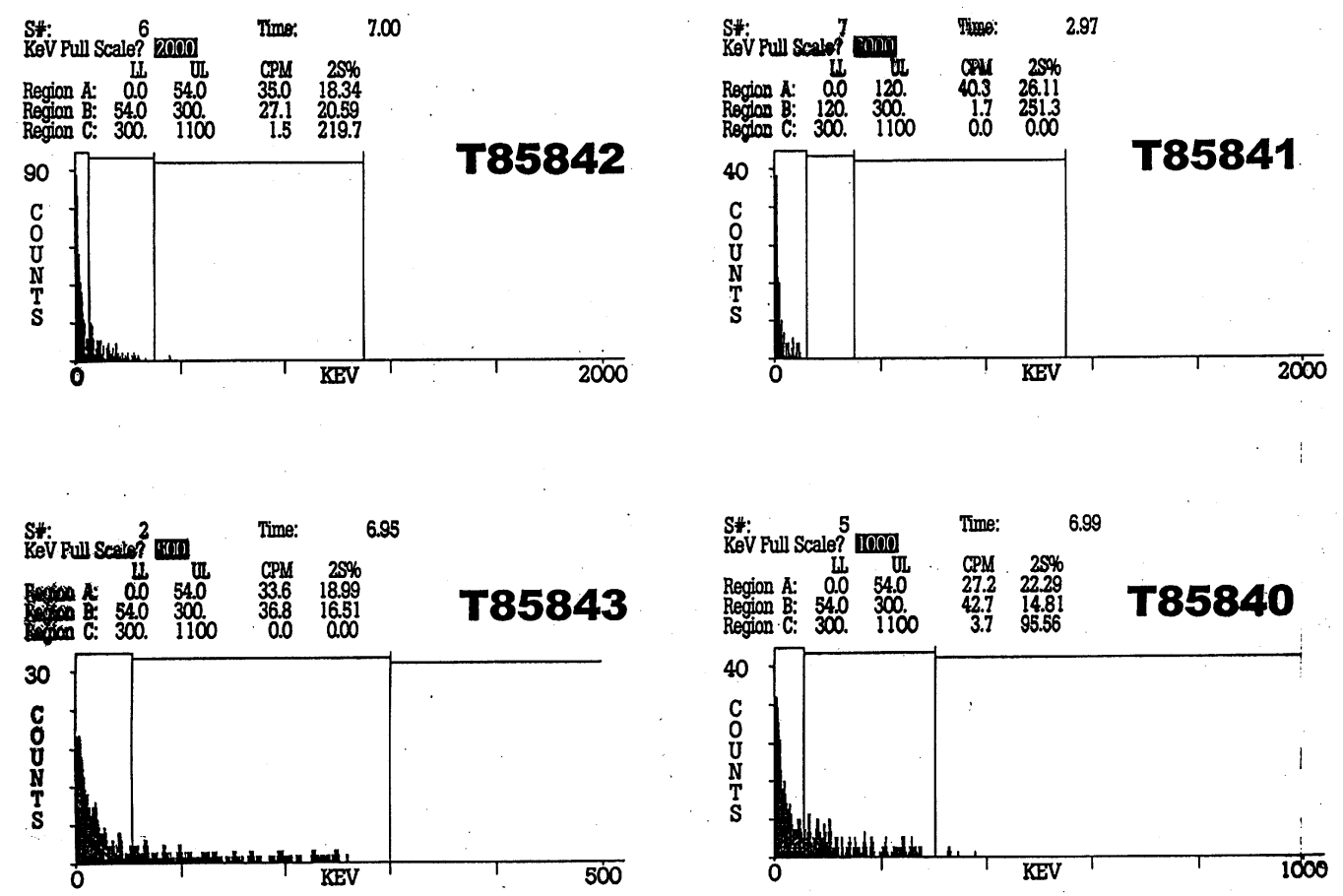

\footnotetext{
Figure 4. Liquid scintillation $\beta$-decay spectra from four intermediate T-phases as described in the text. The spectra each show a low energy Ni-63 component and a long-lived higher energy component attributed to Cs-137.
} 
T85841.sp

CsR

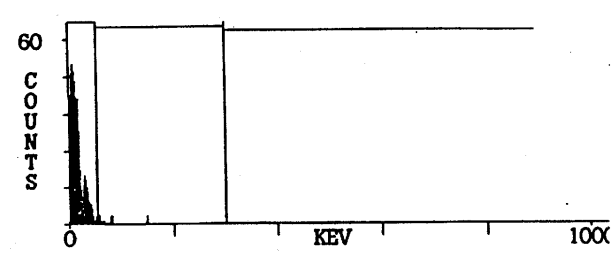

T85841

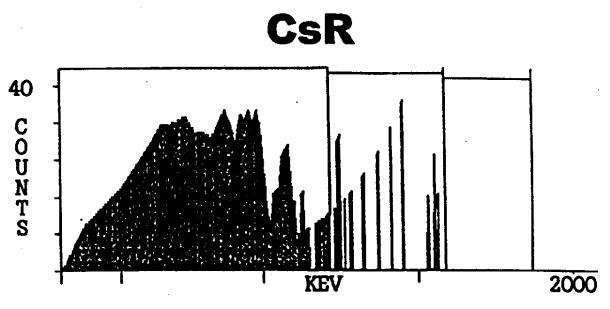

\section{T85842}

CsR

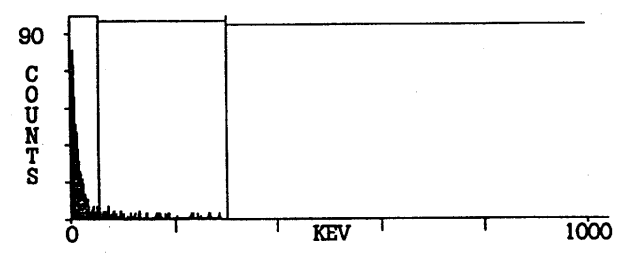

Figure 5. Liquid scintillation $\beta$-decay spectra from three final product phases of the first set of glass samples. The spectra each show only a low energy Ni-63 component. 


\section{B85857}

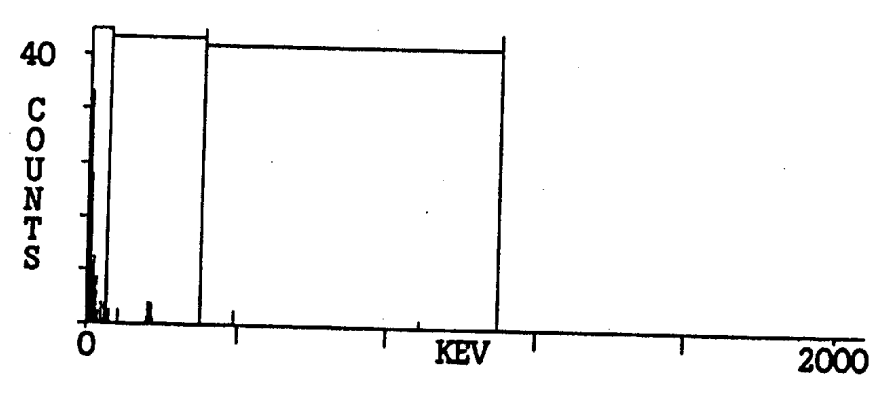

B85859

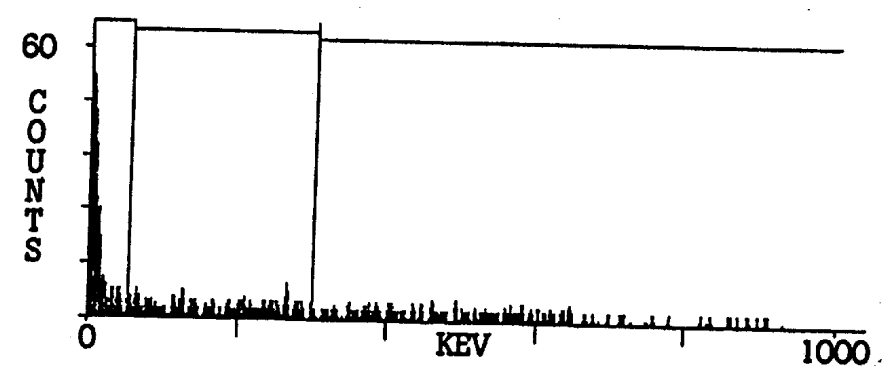

Figure 6. Liquid scintillation $\beta$-decay spectra from two final product phases of the second set of actual DWPF glass product samples. The spectra each show a low energy Ni-63 component and a rapidly decaying Y-90 component. 


\section{SLUDGE TO GLASS DILUTION FACTORS}

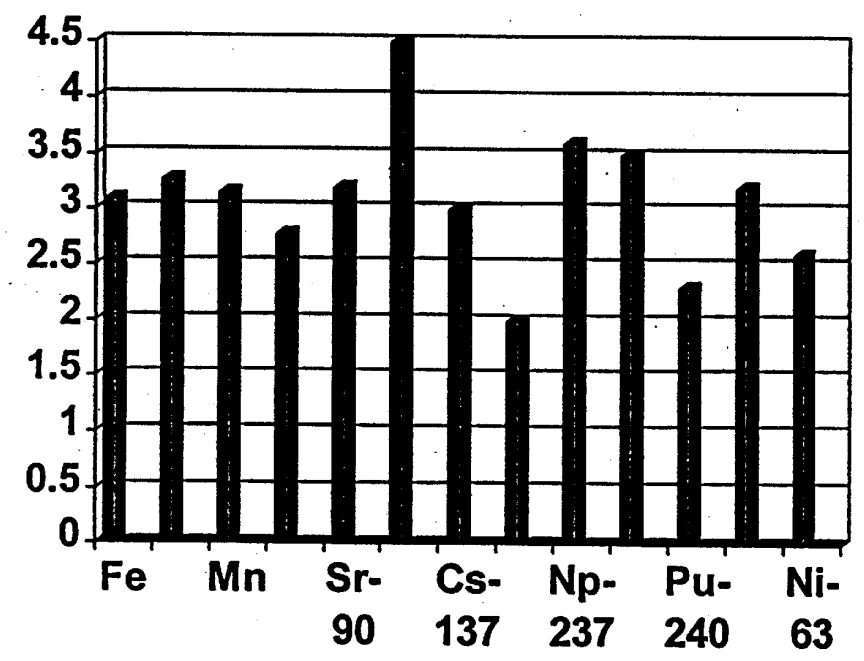

Waste Dilution Factor

\footnotetext{
Figure 7. Sludge to glass waste dilution factors for four stable elements and for $\mathrm{Ni}-63$ and eight other easily measured radionuclides.
} 